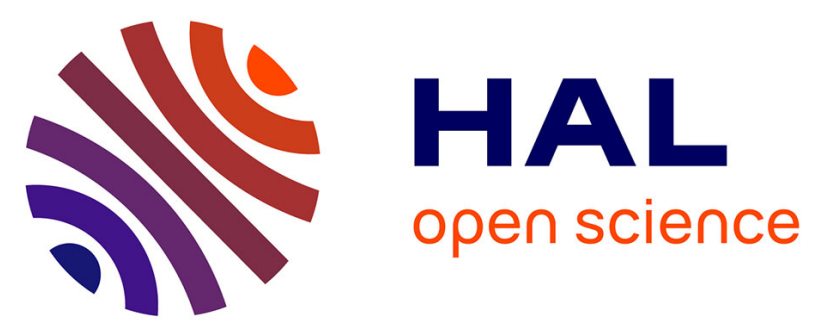

\title{
La rédaction d'une histoire de vie chez les personnes âgées : fondements conceptuels, dimensionnement et proposition d'une échelle de mesure des motivations au récit de vie
}

Samuel Guillemot, Bertrand Urien

\section{To cite this version:}

Samuel Guillemot, Bertrand Urien. La rédaction d'une histoire de vie chez les personnes âgées: fondements conceptuels, dimensionnement et proposition d'une échelle de mesure des motivations au récit de vie. Recherche et Applications en Marketing (French Edition), 2010, 25 (4), pp.25-43. 10.1177/076737011002500402 . hal-00583848

\section{HAL Id: hal-00583848 \\ https://hal.univ-brest.fr/hal-00583848}

Submitted on 6 Apr 2011

HAL is a multi-disciplinary open access archive for the deposit and dissemination of scientific research documents, whether they are published or not. The documents may come from teaching and research institutions in France or abroad, or from public or private research centers.
L'archive ouverte pluridisciplinaire HAL, est destinée au dépôt et à la diffusion de documents scientifiques de niveau recherche, publiés ou non, émanant des établissements d'enseignement et de recherche français ou étrangers, des laboratoires publics ou privés. 
La rédaction d'une histoire de vie chez les personnes âgées : fondements conceptuels, dimensionnement et proposition d'une échelle de mesure des motivations au récit de vie

Version auteurs, Article paru dans Recherche et Applications en Marketing, 2010, 25, 4, $25-43$.

Samuel Guillemot *

Doctorant

IAE de Bretagne Occidentale Brest

Bertrand Urien *

Professeur des universités

IAE de Bretagne Occidentale Brest

* Laboratoire ICI,

IAE Bretagne Occidentale,

12 rue de Kergoat 29238 Brest Cedex 3,

0298017368

samuel.guillemot@univ-brest.fr; bertrand.urien@univ-brest.fr 
La rédaction d'une histoire de vie chez les personnes âgées : fondements conceptuels, dimensionnement et proposition d'une échelle de mesure des motivations au récit de vie

\title{
Résumé:
}

Au cours de cet article nous nous intéressons à un phénomène peu connu en Marketing et qui suscite pourtant un véritable engouement chez les personnes âgées: la rédaction de l'histoire de vie. Alors que la pratique des «écritures du moi » a fait l'objet de nombreuses recherches en gérontologie, en sociologie, et surtout en littérature, aucune recherche marketing n'a porté sur ces comportements spécifiques. L'objet de cet article est d'identifier le concept de récit de vie et de proposer à la communauté académique et managériale une première échelle de mesure des motivations qui le sous-tendent. Deux recueils de données par questionnaires (202 et 508 réponses) ont permis de développer et de valider une échelle de mesure composée de vingt items répartis sur six dimensions (flatter l'ego, réparer l'ego, ne pas être oublié, partager, transmettre et témoigner). Ce papier constitue une contribution au corpus théorique sur les objets spéciaux, et la transmission intergénérationnelle. Il montre que la signification d'un objet spécial ne relève pas uniquement des évocations symboliques qui peuvent se perdre ou être dénaturées mais qu'elles peuvent être explicites et inscrites au cœur même de l'objet.

Mots-clés : Histoire de vie, Personne âgée, Transmission intergénérationnelle, Identité

Legacy writing among the elderly: conceptual bases, dimensioning and a proposed scale for measuring motivations

\begin{abstract}
:
This article concerns a relatively unknown phenomenon in marketing that has become, however, extremely popular among older adults: legacy writing. While the writing of "egodocuments" has been the subject of many studies in gerontology, sociology and, above all, literature, research in marketing has yet to examine its specific components. The purpose of this article is to identify the concept of legacy writing and propose an initial scale to the academic and managerial community for measuring the motivations underlying this practice. Two sets of data collected with questionnaires (202 and 508 responses) have been used to develop and confirm the validity of a scale consisting in twenty items, divided into six dimensions (flattering the ego, mending the ego, being remembered, sharing, transmitting, and bearing witness). This research offers a contribution to the theoretical corpus on special objects and intergenerational transmission. It demonstrates that the meaning of a special object is not exclusively restricted to symbolic references that may be lost or denatured, but others that are explicit and inscribed at the very core of the object.
\end{abstract}

Key-words: Life Story, Aging people, Intergenerational transfer, Identity 


\section{La rédaction d'une histoire de vie chez les personnes âgées : fondements conceptuels, dimensionnement et proposition d'une échelle de mesure des motivations au récit de vie}

\section{INTRODUCTION}

«Vous aimeriez transmettre votre expérience, parfumer vos amours d'un brin d'éternité mais vous n'avez ni le temps ni le courage de prendre la plume. L'écrivain biographe vous écoute. Il s'imprègne de votre parcours, de vos émotions. Le récit naît de vos souvenirs....L'écrivain biographe vous aide à devenir l'auteur du livre qui vous ressemble »1 . Cet extrait emprunté au site web d'un des nombreux biographes privés existant sur le marché illustre l'engouement actuel pour les mémoires et autobiographies. Celui-ci semble plus particulièrement prégnant chez les seniors si l'on se fie aux nombreuses propositions commerciales censées répondre au besoin de mémoire et de transmission dont se font échos les médias qui leur sont destinés. Beaucoup d'individus âgés sont engagés dans la construction mentale d'une histoire de vie cohérente. Si certains d'entre eux passent à l'acte, la plupart envisage de rédiger un jour leurs mémoires sans pour autant concrétiser leur démarche. La réalisation d'une autobiographie est un processus long et difficile, certaines personnes auraient besoin d'un «porte plume», alors que d'autres, simplement d'être guidées. Le marché des services dits «biographiques » est très hétérogène en plein essor et relativement peu structuré. On y déplore ainsi de nombreux abus. Par exemple, certains écrivains privés n'hésitent pas à «livrer» une biographie pour $8000 €$, alors que d'autres rendront une prestation similaire pour $2500 €^{2}$.

En fait, tant du point de vue managérial, qu'académique, c'est un champ d'investigation méconnu en Marketing. Alors que la pratique des «écritures du moi » (biographies, autobiographies, journaux, mémoires, etc.) (Gusdorf, 1991) a fait l'objet de

\footnotetext{
${ }^{1}$ Anne Thomas, ecrivain-biographe.com

${ }^{2}$ Magazine Lire, $\mathrm{n}^{\circ} 353$, mars 2007
} 
nombreuses recherches en gérontologie, en sociologie et surtout en littérature, aucune recherche n'a porté sur ces comportements spécifiques dans notre discipline. Cette recherche s'inscrit plus spécifiquement dans le cadre de l'expérience du vieillissement (Guiot, 2006). Le vieillissement subjectif et la perspective future limitée entraînent des mécanismes d'ajustement dont peuvent naître la recherche d'une continuité de soi et les désirs de préservation et/ou d'extension de soi (Urien et Guiot, 2007). Si le marketing étudie les comportements d'extension, de préservation de soi et de transmission intergénérationnelle (Belk, 1988 ; Wallendorf et Arnould, 1988 ; Price, Arnould et Curasi, 2000 ; Curasi, 2006), ces recherches se focalisent bien souvent sur une préservation et une transmission symbolique du self au travers d'objets «spéciaux ou chéris ». Or la signification de l'objet transmis est fragile et risque d'être transformée par la personne qui va le recevoir (Epp et Arnould, 2006). De façon intéressante, l'écriture de l'histoire de vie permettrait de dépasser ces significations symboliques. S'agit-il dès lors d'une pratique intergénérationnelle visant à transmettre auprès de ses descendants des éléments que l'on juge utile? S'agit-il davantage d'une pratique identitaire qui consiste à mettre en valeur et à préserver une vie riche en souvenirs, en expériences et en significations ? Plus généralement, quelles sont les motivations qui soustendent l'élaboration d'une histoire de vie ? Telles sont les nombreuses questions que peut susciter ce champ d'investigation.

L'objet spécifique de cette recherche est d'identifier le concept de récit de vie et de proposer à la communauté académique et managériale une première échelle de mesure des motivations qui le sous-tendent. Nous limiterons ici nos investigations au segment des personnes âgées, en raison de la fréquence des ces pratiques à ce stade du parcours de vie. Dans un premier temps et à partir d'une revue interdisciplinaire de la littérature, nous proposons une brève approche sociohistorique de la pratique des «écritures du moi », précisons en quoi les personnes âgées sont particulièrement concernées, et identifions la 
nature des différentes motivations qui sous-tendent le récit de vie. Dans un second temps, nous proposons de construire et de valider une échelle de motivations au récit de vie au moyen d'une double étude quantitative. Nous discutons enfin des apports, des implications managériales, des limites mais également des voies d'investigations futures soulevées par notre recherche.

\section{CADRE THEORIQUE DE LA RECHERCHE}

Après avoir retracé les aspects socio-historiques et défini le concept de récit de vie, nous envisagerons les différentes motivations internes telles qu'elles ont été identifiés dans les disciplines sources.

\section{Aspects sociohistoriques}

Les «écritures du moi » se sont développées à partir du $18^{\text {ème }}$ siècle, en parallèle au processus d' "individualisation ». A cette époque, les privilégiés qui ont accès à l'écrit commencent à se soustraire à l'influence des idées dominantes de l'Eglise et de l'Etat et prennent conscience de la singularité de l'individu (Pudal, 2000). Peu à peu, l'effritement du monde traditionnel, l'affaiblissement des contraintes collectives et l'accès à une meilleure éducation entraînent l'individu dans un monde offrant des valeurs, des normes et des pratiques contradictoires. C'est dans cette « ère de l'individu » qui pousse à être performant dans chaque aspect de sa vie, tout en en restant «soi-même», que les écrits personnels s’épanouissent (Delory-Momberger, 2005). Le récit permet de conjuguer ces vies multiples, en sélectionnant puis en tissant des liens entre les évènements. On distingue généralement l'écriture fragmentaire (de type journaux intimes), de l'écriture rétrospective de soi (de type 
mémoire ou biographie). Dans le premier cas, on est dans une démarche de recherche de sens prisée par les adolescents en quête d'une identité, tandis que le second consacre une construction de sens à partir de faits temporels personnels (Pineau et Le Grand, 1993 ; Carron, 2002). Cette dernière approche s'ancre particulièrement bien dans le processus du vieillissement. En effet, à partir de la retraite, les individus sont bien souvent sujets à un retour réflexif sur eux-mêmes et sur leur parcours de vie (Erikson, 1959; Butler, 1963; Castelnuovo-Tedesco, 1980). Cette réflexion approfondie va apporter de nouveaux éclairages sur certains événements de la vie, mettre en valeur des éléments importants que l'on va chercher à préserver et éventuellement à transmettre (Guiot, 2006). D'autant plus, qu'une crainte partagée par les personnes vieillissantes est de voir se disperser après leur mort, souvenirs, expériences et autres significations accumulées au cours d'une vie (Curasi, 2006; Epp et Arnould, 2006).

\section{Les motivations aux récits de vie}

De nombreuses disciplines s'intéressent à l'élaboration des histoires de vie. La sociologie se focalise sur son rôle dans la construction identitaire (Delory-Momberger, 2005), la gérontologie étudie plus spécifiquement son influence dans l'adaptation au vieillissement (Argentin, 2006). Le courant littéraire du genre autobiographique est quant à lui centré sur l'analyse des récits (Lejeune, 1975, 2005). Une synthèse de la revue interdisciplinaire fait apparaitre six motivations génériques :

\section{Flatter l'ego}

La quête de reconnaissance est décrite comme l'un des piliers de l'écriture personnelle. Le récit offre l'occasion de se présenter aux autres (Madelénat, 2008), de mettre 
en valeur un parcours positif et autorise le narrateur à souligner des aspects de sa vie qu'il juge dignes d'intérêts, comme ses réussites, ses compétences, un parcours scolaire ou professionnel, etc. (Rioul, 2003). Poussée à l'extrême, cette démarche peut-être perçue comme une gratification narcissique, les faits étant sélectionnés et orientés de manière à faire l'apologie de soi, jusqu'à se présenter comme « une référence ou un exemple » (Miraux, 2005).

\section{Réparer l'ego}

Outre le fait de flatter son auteur, le récit sert bien souvent d'exutoire à son mal-être. Il ne faut pas négliger le pouvoir libérateur du récit qui permet d'écrire ce qui a été longtemps contenu, de crever l'abcès (Zarca, 2009). De manière analogue, l'histoire de vie offre la possibilité de se défendre d'accusations « injustes » (Hubier, 2003), de donner sa version des faits, corriger ou démentir (May, 1979). Chez les «illustres», Rousseau a été un des premiers, en son temps, à utiliser ses confessions pour justifier sa conduite.

\section{Ne pas être oublié}

Dans les dernières années de sa vie, l'élaboration d'une histoire de vie aide à accepter son sort. En effet, l'analyse approfondie de sa propre vie permet de se présenter comme le héros d'une vie unique et bien remplie (Tarman, 1988) et de faire l'inventaire de ce que l'on veut laisser sur terre, que ce soit des biens matériels ou des expériences de vie (Vercauteren et Hervy, 2003). Dans ce sens, le récit de vie peut avoir pour vocation de survivre à son auteur, car il permet au narrateur de communiquer sur ce qu'il aimerait qu'on retienne de lui et ce qu'il a fait (Cristini et Ploton, 2009). 


\section{Partager}

En Etablissement Hébergeant des Personnes Agées, l'histoire de vie est valorisée comme moyen de communication, vecteur d'échange et de partage (Janvier, 2007) car elle permet de créer un lien social entre les résidents (Billé, 2006 ; Ribes et alii, 2009), entre les résidents et le personnel (Ross, 1990 ; Blanchard, 2006 ; Trueman et Parker, 2006) et parfois même entre les résidents et leurs propres familles (Argentin, 2006). Ainsi, en évoquant son histoire de vie, le narrateur peut chercher à entraîner une forme de complicité avec le lecteur.

\section{Transmettre}

L'histoire de vie est également décrite comme un moyen de transmission qui offre la possibilité de «passer le témoin », elle offre la possibilité de s'inscrire dans une lignée, de faire connaître aux plus jeunes, les membres de la famille aujourd'hui disparus Elle permet ainsi de retrouver le rôle social qui était autrefois dévolu aux anciens, celui de préparer les jeunes générations à l'avenir (Billé, 2002).

\section{Témoigner}

Enfin, l'histoire de vie peut servir à préserver certains éléments de génération en génération. A la différence de la fonction précédente qui s'inscrit dans le cercle familial, la volonté est d'apporter ici une contribution plus large à la mémoire collective. En effet, le narrateur peut, par exemple, mettre en exergue un métier disparu avec l'arrivée des technologies modernes ou devenir historien de son village (Lecarme et Lecarme-Tabone, 1999). Relater des événements dont on fut acteur ou témoin privilégié est un moyen de représenter un destin collectif (Zanone, 2006). Finalement, les histoires de vie permettent d'apporter une contribution à la «connaissance universelle », à destination des sociologues, des historiens ou autres chercheurs de demain. 
Ainsi, la rédaction d'une histoire de vie peut être considérée comme un processus de construction de sens à partir de faits temporels personnels. Le récit peut être centré - ou pas sur le narrateur, être destiné aux contemporains ou à la postérité. Il résulte notamment de motivations profondes que nous avons identifiées lors de notre revue de littérature. Ainsi, flatter l'ego et témoigner sont proposées par le genre littéraire, réparer l'ego est mis en évidence à la fois en littérature et en sociologie. La gérontologie souligne l'importance des motivations de partage, de transmission et celle de ne pas être oublié.

CRÉATION ET VALIDATION DE L'ÉCHELLE DE MESURE DES MOTIVATIONS AU RÉCIT DE VIE

Nous avons commencé par une réflexion approfondie sur le domaine du construit, que nous détaillons par la suite. Ceci nous a amené à choisir une modélisation avec des indicateurs réflexifs. Nous avons donc suivi un certain nombre d'étapes en nous inspirant du paradigme de Churchill (1979) et en prenant en compte les remarques formulées par Rossiter (2002) tout en les adaptant aux outils d'analyse actuelle (équations structurelles). Celles-ci sont résumées dans le tableau $\mathrm{n}^{\circ} 1$ et discutées dans les paragraphes suivants.

<Insérer le tableau $n^{\circ} 1$ - Les étapes de la construction et de la validation de l'échelle de mesure > 
Nature du construit

Pour Rossiter (2002), la définition d'un construit repose sur trois éléments: l'objet (l'élaboration d'une histoire de vie), l'attribut (les motivations) et les évaluateurs (les personnes âgées de 60 ans et plus). Autrement dit, nous cherchons à mesurer les motivations des individus de 60 ans et plus à élaborer des histoires de vie. De manière plus précise, nous allons proposer six variables latentes représentant les différentes dimensions des motivations (flatter l'ego, réparer l'ego, ne pas être oublié, partager, transmettre et témoigner). Afin d'appréhender ces construits, nous leur adjoignons des manifestations observables, comme, par exemple, «j'aimerais écrire pour faire connaitre des aspects de moi dont je suis fièr(e)» pour le construit flatter l'ego.

Se pose dès lors la question cruciale de la relation entre ces variables latentes et les variables observées. Rappelons en effet qu'une erreur sur le sens de causalité entre une variable manifeste et un construit latent lors de la modélisation entraine une procédure de purification des items inappropriée (Diamantopoulos et Winklhofer, 2001 ; Jarvis et alii, 2003 ; Crié, 2005). Les articles de Jarvis et alii (2003) et Podsakoff et alii (2003) s'inscrivent au cœur du débat « réflexif $v s$ formatif ». Après lecture de contributions récentes issues des numéros spéciaux de Psychological Methods (2007; $\left.\mathrm{n}^{\circ} 12,2\right)$ et de Journal of Business Research $\left(2008 ; \mathrm{n}^{\circ} 61\right)$, notre réflexion nous a conduit à choisir un modèle de mesure réflexif (les variables observées sont des effets des construits), ceci pour plusieurs raisons :

Premièrement, en suivant Howel et alii (2007a) et Bagozzi (2007), nous pensons, à la différence de Podsakoff et alii (2003), que les construits ne sont pas formatifs ou réflexifs par nature et que la conception du modèle de mesure est d'avantage un choix épistémologique du chercheur plutôt qu'une caractéristique du construit lui-même. Nous nous plaçons dans notre étude dans un paradigme «réaliste»(Howell et alii, 2007a), en ce sens que le construit 
mental considéré existe en dehors de ses mesures. D'autre part, l'utilisation d'indicateurs réflexifs est à privilégier, compte tenu des limites inhérentes aux construits formatifs, formulées de façon explicite dans plusieurs contributions récentes (Diamantopoulos, 2006 ; Bollen, 2007 ; Franke et alii, 2008 ; Howel et alii, 2007a; Diamantopoulos et alii, 2008). Parmi celles-ci, le fait que le terme d'erreur ne représente pas une erreur de mesure mais le surplus de signification du construit qui n'est pas capturé par ses indicateurs, est très pénalisant, car dans la pratique, il est impossible de détecter toutes les causes possibles d'un construit (Howel et alii, 2007b). Les mesures formatives seraient également sujettes à des confusions d'interprétations (ibid)

Deuxièmement, Wilcox et alii (2008) suggèrent qu'une même liste d'item peut être conceptualisée de manière formative ou réflexive selon les instructions que le chercheur doit donner aux répondants. Si la formulation des questions se base sur des actions passées «j'ai écris ma biographie parce que...», les indicateurs sont à priori formatifs de l'écriture de vie (ce sont des causes). En revanche, si l'on évoque des actions futures «J'aimerais écrire ma biographie pour...», le jugement se base sur des actions hypothétiques et les indicateurs deviennent des reflets du construit (Wilcox et alii, 2008). Dans le premier cas, on obtient un construit «objectif», tandis que dans le second cas, des indicateurs réflexifs donnent un construit de nature subjective correspondant aux construits de nature psychologique que l'on utilise fréquemment en comportement du consommateur (Bollen, 2007). 


\section{Création et Validation des items}

Nous avons réalisé une étude qualitative auprès de 8 individus ayant rédigé une histoire de vie et 8 autres projetant de le faire. L'étude qualitative, non développée ici, a permis de retrouver les 6 motivations identifiées précédemment. Nous avons extrait du corpus 96 verbatim caractérisant les 6 types de motivations. A partir de ces verbatim et de la littérature, et en tenant compte des précisions méthodologiques énoncées précédemment, nous avons généré une liste de 77 items représentant des reflets de chaque type de motivation.

Afin de nous assurer de la validité de contenu et réduire la liste initiale des items, les propositions ont été soumises à une liste de cinq experts, chercheurs confirmés en comportement du consommateur, habitués à construire et/ou à manipuler des échelles de mesure. Suivant la procédure préconisée par Jolibert et Jourdan (2006), les experts ont reçu une définition du construit et de ses composantes. Ils ont dû apprécier chacun des items comme «très », «assez » ou «peu » représentatifs des dimensions auxquelles ils étaient rattachés. Les experts se sont également prononcés sur la clarté des propositions, certains items ayant ainsi été reformulés. Seuls les items jugés «très représentatifs » par une majorité d'experts ont été sélectionnés à condition de ne pas avoir été jugés peu représentatifs par l'un des autres experts. Finalement 36 items (soit 46,75\% du total initial) ont été retenus (Annexe A1).

Méthodologie des collectes des données

Deux collectes de données ont été réalisées. La première a été conduite pendant l'été 2009 et nous a permis de purifier l'échelle de mesure à l'aide d'analyses factorielles en composantes principales exploratoires réalisées avec le logiciel SPSS. La seconde a été 
conduite à l'automne de la même année et a servi de support à une analyse factorielle confirmatoire avec le logiciel EQS. Les répondants ont été invités à se prononcer sur leur degré d'accord avec les libellés placés dans un ordre aléatoire (Annexe 1) et proposés sur une échelle de Likert en 5 points allant de «pas du tout d'accord » à «tout à fait d'accord ». Le nombre d'échelons a été fixé à cinq car les personnes les plus âgées ne sont pas à l'aise lorsque le format de réponse présente trop de nuances (Mezred et alii, 2006). Les questionnaires ont été distribués in situ, sur les lieux où l'on a le plus de chance de rencontrer des personnes concernées par l'étude (maison de retraite, clubs et associations de retraitées, universités du temps-libre, etc.). Etant donné le caractère personnel de certaines questions, nous avons privilégié une collecte des données auto-administrée. Au total, 710 réponses exploitables ont été récoltées, respectivement 202 lors de la première collecte et 508 lors de la suivante. Les personnes interrogées sont toutes âgées de plus de 60 ans. Ce seuil a été retenu car de nombreux spécialistes, tant du domaine de la santé, de la psychologie, que du marketing, s'accordent à dire qu'avec la retraite, il marque le début du vieillissement (Bresay, 2004 ; Fry, 1990 ; Guiot, 2006). L'âge moyen des répondants est de 71,28 ans (écart-type 8,6)

pour la première collecte et de 68,80 ans (écart-type 6,84) pour la seconde. Les deux échantillons sont composés respectivement de $56 \%$ et $64 \%$ de femmes Le niveau d'étude est très hétérogène, ce qui témoigne de la diversité sociale de nos échantillons (Annexe A2).

\section{Première collecte de données et purification des items}

Après avoir écarté les questionnaires présentant trop de données manquantes et vérifié l'absence d'outliers (Hair et alli, 2008) la première collecte de données nous offre 202 données exploitables. L'analyse de la matrice des corrélations des 36 variables (non reproduite ici), nous montre que $94 \%$ des corrélations sont significatives. Compte tenu de ce 
niveau d'inter-corrélations, il n'est pas étonnant d'obtenir un score de 0,89 au test MSA. Ce qui signifie que les indicateurs partagent quelque chose en commun et justifie un processus de réduction d'information (Kaiser et Rice, 1974). Une série d'analyse en composantes principales nous a permis d'identifier un ensemble de dimensions latentes. Les composantes étant supposées corrélées, nous avons utilisé une rotation oblique Oblimin des axes factoriels pour en faciliter l'interprétation. Seuls les facteurs dont les valeurs propres associées sont supérieures à 1 ont été retenus. Enfin, nous avons supprimé les items ne s'agrégeant sur aucun axe, ou au contraire saturant de manière comparable sur plusieurs facteurs.

Selon ces critères, 6 ACP ont été nécessaires pour aboutir à une solution stable. A ce stade, les 14 items restant s'agrégent sur 4 facteurs. Les items générés représentant les dimensions transmettre et flatter l'ego ont disparu lors de l'épuration de l'échelle (Annexe A1). Pour les premiers, la formulation des items est remise en cause. Dans un souci de généraliser les énoncés pour éviter les cas particuliers nous avons écarté les mots issus du vocabulaire familial pour ne pas exclure les répondants sans enfant. Or les items ainsi formulés sont devenus trop généraux et ne faisaient pas explicitement référence aux valeurs, aux racines et à l'histoire familiale qui sont, selon la littérature et nos entretiens qualitatifs, les éléments qui sont le plus fréquemment transmis. Pour la composante flatter l'ego c'est la définition soumise aux experts qui est remise en cause, celle-ci insistait d'avantage sur le caractère narcissique (pour parler des choses que j'ai pu faire) que sur l'aspect reconnaissance qui entre également en compte (pour faire connaître des aspects de moi que les autres ne connaissent pas forcément). Fort de ces remarques, nous générons des nouveaux items pour ces deux composantes ( 9 au total). Ces items ont été validés par trois nouveaux experts, nous les avons ajoutés aux 14 autres afin de procéder à une nouvelle collecte de données (Annexe A1). 
Seconde collecte de données et émergence d'une structure factorielle

La seconde collecte de données nous offre 508 données exploitables. Après avoir vérifié l'absence de données aberrantes, nous procédons à une nouvelle purification des items selon les règles définies dans l'étape précédente. L'analyse restitue 6 facteurs pour 20 items. Seules les valeurs propres associées aux cinq premiers facteurs sont supérieures à 1 . Le « Scree-test » montre une première cassure après la cinquième composante (valeur propre 1,07 ) et une seconde moins nette après la sixième (valeur propre 0,93 ). Nous retenons la sixième dimension car elle se justifie d'un point de vue théorique. D'autant plus qu'elle apparaît sur des sous-échantillons tirés de manière aléatoire. Au final, l'échelle de mesure se compose de 6 dimensions comportant de 3 à 4 items chacune. Les coefficients $\alpha$ s'échelonnent de 0,79 pour la dimension partager à 0,83 pour la dimension témoigner (Tableau $\mathrm{n}^{\circ}$ ).

\section{< Insérer Tableau $\mathbf{n}^{\circ} \mathbf{2}$ - Structure factorielle finale des 20 items >}

Validation de la structure factorielle

L'objectif est maintenant de valider la structure factorielle qui vient d'émerger à l'aide de la modélisation par équations structurelles. L'analyse factorielle confirmatoire (AFC) suppose une distribution normale des données. Le test du coefficient de Mardia (G2, P) montre une absence de normalité des données. Il restitue une valeur de 85 (normalized estimate $=32$, bien au-delà de la limite acceptable fixée à 3 par Bentler (2005). En réalité, dans le domaine des sciences sociales, les données suivent rarement une distribution normale. La statistique normée de Satorra et Bentler (1988), disponible sous EQS sous l'appellation 
«robust» produit des coefficients de significativité corrigés en fonction de l'intensité de la non-normalité.

Le modèle testé est composé des 6 variables latentes autorisées à covarier librement. Celui-ci produit de bons indices d'ajustement compte tenu du degré de complexité et du nombre d'observations. Pour un modèle comportant 20 items et 508 observations, le CFI doitêtre supérieur à 0,92 et le RMSEA sous le seuil de 0,07 (Hair et alii., 2008). Ici, c'est le cas ; outre le $\chi^{2}$ de 340 pour 155 degrés de liberté ( $\mathrm{p}=0,00$ ), la valeur du CFI est de 0,95 et celle du RMSEA de 0,05 (intervalle de confiance de 90\% : [0,042-0,056]). Etant donné le degré élevé des corrélations entre les construits, il nous a semblé important de tester des modèles alternatifs. Plus particulièrement le modèle à cinq facteurs qui avait émergé initialement. Les indices d'ajustement de ce dernier sont sous les seuils communément admis et par conséquent inférieurs à ceux du modèle à 6 facteurs $\left(\chi^{2}=557\right.$; ddl $=160(\mathrm{p}=0,00) ; \mathrm{CFI}=0,90$; RMSEA $=0,07)$. De la même manière, plusieurs autres modèles ont été testés, par exemple le modèle unidimensionnel $\left(\chi^{2}=1194 ; \mathrm{ddl}=170(\mathrm{p}=0,00)\right.$; CFI $=0,74$; RMSEA $\left.=0,11\right)$, mais aucun ne produit d'aussi bons indices d'ajustement que le modèle à six dimensions. La structure factorielle à six dimensions est donc la meilleure et peut être retenue.

Fiabilité, validités convergente et discriminante du modèle de mesure

Nous étudions dans cette étape les qualités psychométriques de l'instrument de mesure avec l'examen de la fiabilité, des validités convergente et discriminante. La validité de contenu a déjà été vérifiée, puisque les items ont été sélectionnés par des experts. La validité nomologique sera examinée dans la section suivante.

La fiabilité est vérifiée lorsque tous les indicateurs mesurent le même construit de manière pas trop inégale. Ici, elle est assurée, les coefficients rhô de cohérence de Jöreskog 
( $\rho$ ) sont tous supérieurs à 0,7 (tableau 3) qui constitue un seuil communément admis (Fornell et Larker, 1981).

La validité convergente correspond à la capacité d'une mesure à fournir des résultats proches de ceux d'autres mesures du même trait. Elle est vérifiée lorsque : (1), le test z associé à chacune des contributions factorielles est > 1,96 (lien significatif entre une variable latente et ses indicateurs); et (2), chaque indicateur partage plus de variance avec son construit, qu'avec le terme d'erreur qui lui est associée (Steenkamp et Van Trijp, 1991). Dans les faits, cette condition est remplie si la variance moyenne extraite $\left(\rho_{\mathrm{vc}}\right)$ pour chaque dimension est $>$ à 0,5 . Toutes les variables latentes remplissent ces deux conditions (tableau $3)$.

\section{<Insérer Tableau $n^{\circ} 3$ - Evaluation de la fiabilité et de la validité convergente >}

La validité discriminante est assurée lorsque que deux construits différents théoriquement sont bien distincts empiriquement. Ainsi, il faut que les corrélations entre deux construits soient inférieures à la racine carrée de leurs $\rho_{\mathrm{vc}}$, ce qui est le cas ici (tableau 4). A noter néanmoins que certaines corrélations sont élevées, comme par exemple entre les composantes réparer l'ego et ne pas être oublié $(0,70)$. Toutefois les indicateurs d'une même dimension partagent plus de variance entre-eux qu'avec ceux de l'autre dimension.

<Insérer Tableau $\mathbf{n}^{\circ} 4$ - Evaluation de la validité discriminante et corrélations entre les construits> 
Les corrélations entre les construits étant toutes importantes et significatives (de 0,50 et 0,70 ), nous avons effectué une analyse factorielle exploratoire de second ordre. Celle-ci fait apparaitre un seul facteur d'ordre supérieur. L'analyse confirmatoire produit de bons indices d'ajustement $\left(\chi^{2}=381 ; \mathrm{ddl}=164(\mathrm{p}=0,00) ; \mathrm{CFI}=0,95 ;\right.$ RMSEA $\left.=0,05\right)$. Les loadings standardisés entre les construits de premier ordre et le facteur d'ordre supérieur sont tous significatifs et supérieurs à 0,7 . Le modèle avec un seul facteur de second ordre est donc accepté (tableau 5). Cela signifie que les six motivations partagent bien un concept commun qui serait une motivation globale à élaborer une histoire de vie. Sur le plan théorique, cela apparaît en adéquation avec une conception hiérarchique de la motivation (Vallerand, 1997, 2000 ; Vallerand et Miquelon, 2009). La motivation globale au récit de vie s'exprimerait sous formes de 6 motivations contextuelles (témoigner, transmettre, etc....). Ici encore, nous suivons Howel et alii (2007a) en considérant que cette motivation globale existe indépendamment de sa mesure. Les variables latentes de premier ordre, représentant les différentes motivations, constituent alors des reflets du facteur de second ordre.

\section{<Insérer Tableau $\mathrm{n}^{\circ} 5$ - Analyse factorielle de second ordre>}

Quant à la validité nomologique, elle est assurée si l'on constate empiriquement qu'un individu avec des motivations au récit de vie a effectivement l'intention d'en réaliser un. En effet, elle vise à confirmer la définition du construit à travers l'examen des corrélations entre le construit et d'autres manifestations qui lui sont intrinsèquement attachées - de manière quasi tautologique - (Jolibert et Jourdan, 2006). L'intention d'écrire une histoire de vie à été opérationnalisée à l'aide d'une mesure mono-item. Cela est suffisant si le construit est 
conceptualisé de façon concrète et unique par le répondant (Rossiter, 2002 ; Bergkvist et Rossiter, 2008), ce qui est le cas des intentions de comportements (Jarvis et alii, 2004). En nous aidant des entretiens semi-directifs réalisés (non traités ici) nous avons pu distinguer différents stades spécifiques relatifs à l'engagement dans la rédaction d'une l'histoire de vie : non intéressé, intéressé mais n'envisage pas d'écrire sa vie, envisage d'écrire un jour sa vie, fortement intéressé par le passage à l'écriture ou déjà engagé dans le processus d'écriture. Ce sont ces modalités qui ont été proposées aux répondants de l'étude quantitative. Afin d'éviter une corrélation artificielle liée à l'ordre d'administration des questions, deux autres échelles de mesure ont été insérées entre les items de mesure des motivations au récit de vie et la mesure de l'intention d'écrire une histoire de vie. D'autre part, bien que l'échelle ainsi élaborée ne soit pas métrique, les données peuvent être estimées dans un modèle d'équations structurelles car les modalités reflètent un degré d'engagement croissant (Byrne, 2006). Dans pareil cas, l'estimation par le maximum de vraisemblance avec les indices corrigés de Satorra et Bentler (1988) garantit des résultats fiables (DiStefano, 2002 ; Bentler, 2005).

Les résultats obtenus indiquent une corrélation significative entre les deux construits ( $\mathrm{r}$ $=0,50$; test $\mathrm{z}=10,16$ ), validant ainsi nomologiquement l'échelle de mesure.

\section{APPORTS, IMPLICATIONS MANAGÉRIALES, LIMITES ET VOIES DE RECHERCHE}

\section{Les apports}

Notre étude avait pour ambition d'aboutir à une identification du concept de récit de vie, d'en cerner son dimensionnement et de proposer une première échelle de mesure des motivations au récit de vie (Annexe A3). A notre connaissance, il s'agit de la première recherche théorique et empirique sur le sujet en comportement du consommateur. A partir 
d'une revue de littérature et de deux collectes de données quantitatives, nous avons élaboré une échelle fiable et valide mesurant les motivations au récit de vie de la personne âgée. Celle-ci présente 20 items qui se répartissent sur six dimensions (flatter l'ego, réparer l'ego, ne pas être oublié, partager, transmettre et témoigner).

Cette échelle n'est qu'une étape dans la compréhension du concept de récit de vie, elle permettra d'appréhender empiriquement les relations qu'entretiennent les motivations au récit de vie avec d'autres variables qui apparaissent dans le réseau nomologique de ce concept. Par ailleurs, cette recherche permet d'enrichir la compréhension des « tactiques » (Price, Arnould et Curasi, 2000) d'extension, de préservation de soi et de transmission intergénérationnelle des personnes âgées (Curasi et alii, 2004 ; Curasi, 2006). Le récit de vie, quel que soit son support (Livre, CD etc...) apparaît alors comme une nouvelle forme d'objet spécial dont la partie symbolique se perd au profit d'une fonction explicite. Rappelons ici que l'objet «spécial » ou «chéri » ( alliance, stylo ayant appartenu au grand père etc.) sert de « véhicule » à 1'histoire familiale et individuelle (Curasi et alii, 2004 ; Bergadaà et Urien, 2008) et possède un certain nombre de fonctions qui s'apparentent à celles que nous venons d'identifier pour l'histoire de vie : il a valeur de reconnaissance, il permet de se remémorer des moments de vie, de témoigner d'évènements particuliers, de rappeler des compétences et des capacités spécifiques (Price et alii, 2000). Il a également une valeur thérapeutique, et sert notamment de point d'ancrage et de stabilité (Wapner et alli, 1990). Il permet enfin de transmettre les valeurs individuelles et familiales. Lorsqu'il est donné comme un cadeau ou un legs à une personne qui saura en préserver le sens, il constitue un moyen de ne pas être oublié (Price et alii, 2000) et est un vecteur de transmission intergénérationnelle (Goldberg, 2009). Mais si le récit de vie peut-être considéré comme un objet spécial, il n'en possède pas moins une spécificité importante. La transmission de l'objet spécial est souvent considérée comme une transmission symbolique du self, l'information transmise étant alors réduite à des 
souvenirs fugaces, générés par l'objet, une fois la personne disparue. Or, le récit de vie va bien au-delà et propose la transmission d'un résumé explicite du self, plus ou moins fidèle, parfois idéalisé. Le caractère explicite de l'information contenue dans le récit de vie confère alors à ce dernier un rôle majeur dans la transmission de la mémoire intergénérationnelle ; et permet vraisemblablement de pallier pour partie, cette fragilité de la signification de l'objet transmis, soulignée en introduction (Epp et Arnould, 2006).

\section{Implications managériales}

Si le marché des services biographiques est à ce jour un marché de niche, le potentiel de développement est néanmoins réel. En effet, lors de la seconde collecte de données (508 individus), $12,8 \%$ des personnes interrogées ont déjà où écrivent actuellement leurs souvenirs. Et plus de 50\% y ont déjà pensé mais sont rebutées par l'ampleur de la tâche, si bien que 42\% se disent intéressées par les services d'apprentissage (18\% pour les services de coproduction $^{3}$ ). Le marketing a ici un rôle fondamental à jouer. S'il réussit à faire en sorte qu'une partie des individus intéressés par la démarche biographique, passe à l'action, notre discipline jouera un rôle important dans la transmission de la mémoire intergénérationnelle. En ce sens, certains anthropologues, constatant une indifférence croissante à l'égard du culte des morts, prédisent l'édification de nécropoles d'un nouveau genre. De véritables «monuments psychiques », les mnémothèques (Larcher, 1971) permettraient de conserver une trace de la mémoire de ceux qui ont vécu plutôt que celle du corps ou des restes physiques (Barreau, 1992). Il y a vraisemblablement ici des gisements de nouveaux services à élaborer en direction des seniors.

\footnotetext{
${ }^{3}$ Comme les biographes privés. On parle ici de «co-production », car le consommateur fournit la matière première (ses souvenirs) et le prestataire les compétences.
} 
D'autre part, l'identification des différentes motivations mises en évidence va permettre aux prestataires de service de segmenter leurs offres, car il est bien évident qu'une personne qui souhaite avant tout transmettre l'histoire de sa famille n'a pas les mêmes besoins et attentes qu'une autre qui souhaite principalement témoigner de son expérience du racisme par exemple. Fondamentalement, les services d'apprentissage des compétences (ateliers, coaching, etc.) sont adaptés lorsque le processus est aussi important que le résultat, comme par exemple, lorsque le récit est guidé par une volonté de réparer l'ego où le processus d'écriture conduit à la « guérison ». Au contraire, les services de « co-production » sont plutôt adaptés lorsque l'histoire est plus importante que le processus qui a contribué à la créer. Par exemple, lorsque le récit est destiné à la communauté (transmettre et témoigner), l'idée sous jacente est de servir les autres, peu importe comment on y arrive.

Il convient bien évidemment de considérer l'éthique d'une telle pratique. Nous avons en effet précisé, au début de cet article, qu'une même prestation pouvait être facturée du simple au triple. N'oublions pas que l'éthique d'un manager s'appuie sur une conscience morale, le suivi d'un éventuel cadre légal et déontologique dans l'exercice de son activité, et sur la responsabilité de ses actes (Katz et Marshall, 2003 ; Bergadaa, 2004). L'utilisation de cette échelle doit donc être réalisée avec précautions en se préoccupant du bien être du consommateur âgé et non pas en profitant de sa vulnérabilité.

\section{Les limites et voies de recherche futures}

Plusieurs limites peuvent être formulées. L'une d'entre elles concerne la validité externe de l'échelle. Celle-ci a été en effet validée sur un échantillon de personnes âgées. Nos résultats constituent une première étape, l'échelle de mesure devant être confrontée à d'autres données représentatives de la population afin d'en accroître sa validité. Notre second 
échantillon était en effet constitué majoritairement de personnes fréquentant les Universités du Temps Libres, qui sont plus éduquées que la moyenne de la population française. On peut se demander également si le modèle biographique français est transposable à d'autres cultures. Chaque culture s'inscrit dans des représentations du soi bien différentes, avec ses propres concepts pour penser et dire les expériences personnelles, familiales et culturelles. Selon Roche (2007), par exemple, l'autobiographie est présente dans les pays du Maghreb, mais elle se traduit le plus souvent par un mélange de réalité, de fiction et de poésie. D’un point de vue plus général, une extension internationale de cette recherche à d'autres contextes culturels, permettrait d'étudier à la fois la stabilité des différentes motivations au récit de vie identifiées ici, et les types de services biographiques existants.

Dans cette étude, nous n'avons pas examiné les effets de cohorte (Meredith et Schewe, 1994 ; Zemke, Raines et Filipczak, 2000). Les évènements socio-historiques survenus pendant les années où s'opèrent les choix de vie (15-25 ans) forment les attitudes et les comportements d'une génération (Préel, 2000). Ainsi la cohorte 1920-1930 qui a vécu et participé à la guerre n'a pas les mêmes choses à raconter et à transmettre que la cohorte 19451955 qui a grandi avec la croissance économique (Lalive d'epinay et Cavalli, 2009). Toutefois, nous pensons que la différence entre générations ne se fera pas dans la nature des motivations mais dans le contenu de l'histoire elle-même. Ce point reste néanmoins à approfondir.

Par ailleurs, nous ne nous sommes pas interrogés sur les raisons qui font préférer une transmission écrite à une transmission orale (Martin Sanchez, 2003). Ainsi notre approche «fonctionnaliste» des histoires de vie et son pendant méthodologique qui consiste à interroger la personne sur ses motivations à élaborer des histoires de vie, peut faire l'objet d'une critique. Cette perspective privilégie les motivations conscientes et intentionnelles des histoires de vie et ne met pas l'accent sur le sens donnés par les individus à leur 
comportement. Une approche compréhensive de la pratique d'écriture de sa vie est dans ce sens une direction de recherche à privilégier.

Il convient maintenant de s'intéresser à d'autres variables pouvant affecter le comportement biographique comme le sentiment d'efficacité personnelle (Bandura, 1986), la générativité (McAdams et de St. Aubin, 1992; Urien et Kilbourne, 2007, 2011), ou encore la réminiscence (Webster, 1993, Haber, 2006). Tant Price et alii (2000), que Curasi et alii (2004) présentent la transmission d'objet spécial comme un désir de préserver le soi au-delà de la mort, une forme d'immortalité symbolique. Ces auteurs présentent toutefois l'échéance ultime de façon indifférenciée (Urien, 2003). Or il existe différentes attitudes face à la mort qui génèrent de l'anxiété ou de l'acceptation. De façon plus précise, Florian et Kravetz (1983) et Florian et Snowden (1989) présentent une conception multidimensionnelle de l'anxiété face à la mort (perte de l'accomplissement de soi, annihilation de soi, perte d'identité sociale, conséquences pour les proches, conséquences transcendentales, punition après la mort). Une question de recherche importante serait l'impact du type d'attitude face à la mort sur la nature et l'intensité des motivations au récit de vie

Une autre piste de recherche consiste à confronter les tendances biographiques des différentes générations. Ainsi, le même type de démarche est envisageable pour comprendre la quête identitaire des adolescents appliquée aux journaux intimes et aux blogs, ou pour comprendre les phénomènes de réseaux sociaux sur internet de type facebook par exemple. La production de soi comme technique relationnelle (Cardon, Delaunay-Teterel, 2006) est un champ d'investigation d'actualité. Le champ d'application des services biographiques peut également s'étendre : par exemple la biographie patronale (d'Almeida et Merran-Ifrah, 2005) ou plus généralement celle des entreprises, comme en témoigne cet article d'un grand quotidien régional ${ }^{4}:$ "En temps de crise, l'Histoire est une valeur sûre. Biographe, Hélène

\footnotetext{
${ }^{4}$ Ouest-France, Novembre 2008
} 
raconte dans ses livres de véritables sagas économiques. Dans la région, nombre de chefs d'entreprises lui font appel ». 


\section{Annexe A1 : Les items générés par les auteurs et retenus par les experts}

Le tableau suivant présente les items générés retenus par les experts :

-Le numéro correspond à la place de l'énoncé dans l'échelle. Par exemple l'item «pour dire à mes proches ce que j'aimerais que l'on retienne de moi » de la dimension ne pas être oublié a été administré respectivement en $15^{\text {ème }}$ et en $8^{\text {ème }}$ position lors des deux collectes. -Le sigle «E $\mathrm{E}$ signifie que l'item a été éliminé suite aux analyses factorielles en composantes principales.

-Les items précédés d'un astérisque (*) ont été générés et administrés lors de la seconde collecte.

\begin{tabular}{|c|c|c|c|c|}
\hline J'aimerais écrire une histoire de vie ... & \multicolumn{2}{|c|}{$1^{\text {ère }}$ collecte } & \multicolumn{2}{|c|}{$2^{\text {nd }}$ collecte } \\
\hline \multicolumn{5}{|l|}{ Ne pas être oublié } \\
\hline - pour mettre en valeur ce que j'aimerais que l'on retienne de moi & 30 & $\mathrm{E}$ & & \\
\hline -afin de sélectionner ce que j'aimerais laisser pour la postérité & 17 & $\mathrm{E}$ & & \\
\hline $\begin{array}{l}\text {-car il est important pour moi de savoir comment mes proches se rappelleront de moi } \\
\text { après ma mort }\end{array}$ & 9 & $\mathrm{E}$ & & \\
\hline - pour dire à mes proches ce que j'aimerais que l'on retienne de moi & 15 & & 8 & \\
\hline -car j'estime que laisser une trace de soi permet d'atteindre une forme d'immortalité & 6 & $\mathrm{E}$ & & \\
\hline - pour que mes proches se rappellent de moi après ma mort & 34 & & 4 & \\
\hline -parce qu’écrire sa vie permet de ne pas être oublié & 12 & $\mathrm{E}$ & & \\
\hline - pour que l'on se souvienne de ce que j'ai fait après ma mort & 20 & & 12 & \\
\hline \multicolumn{5}{|l|}{ Témoigner } \\
\hline -pour permettre aux générations futures de comprendre comment le monde a évolué & 27 & $\mathrm{E}$ & & \\
\hline $\begin{array}{l}\text { - pour laisser des traces sur la façon dont nous vivons, afin que les générations } \\
\text { futures puissent reconstituer notre mode de vie }\end{array}$ & 3 & & 5 & \\
\hline $\begin{array}{l}\text { - pour laisser un témoignage car cela permet de créer une sorte de patrimoine qu'on } \\
\text { lègue aux générations futures }\end{array}$ & 13 & & 9 & \\
\hline $\begin{array}{l}\text {-parce que je pense que livrer ses réflexions sur un aspect de la société peut } \\
\text { permettre aux générations futures de mieux comprendre le monde dans lequel ils } \\
\text { vont vivre }\end{array}$ & 29 & $\mathrm{E}$ & & \\
\hline $\begin{array}{l}\text {-parce que laisser un témoignage sur son époque, c'est un peu comme un cadeau que } \\
\text { l'on fait aux générations futures }\end{array}$ & 31 & $\mathrm{E}$ & & \\
\hline $\begin{array}{l}\text {-parce qu'il y a certaines choses qui doivent-être préservées et transmises de } \\
\text { génération en génération }\end{array}$ & 33 & $\mathrm{E}$ & & \\
\hline - pour préserver certains aspects de ma culture qui sont en train de disparaître & 24 & & 13 & \\
\hline -parce qu'il est nécessaire de sauver certaines choses de l’oubli & 4 & $\mathrm{E}$ & & \\
\hline - pour noter quelque part certaines choses du passé qui ne doivent pas être perdues & 18 & & 16 & \\
\hline \multicolumn{5}{|l|}{ Partager } \\
\hline -pour créer une forme de complicité avec les lecteurs & 19 & & 6 & \\
\hline -pour élargir le cercle de mes connaissances & 2 & & 10 & \\
\hline -pour créer des liens avec certaines personnes & 14 & & 14 & \\
\hline $\begin{array}{l}\text {-parce qu'il est important de raconter des moments difficiles de l'existence, pour } \\
\text { montrer à ceux qui vivent la même chose, qu'ils ne sont pas seuls }\end{array}$ & 16 & $\mathrm{E}$ & & \\
\hline \multicolumn{5}{|l|}{ Réparer l'ego } \\
\hline -pour m'aider à clarifier une période de ma vie & 7 & & 3 & \\
\hline
\end{tabular}


-pour donner ma version des faits sur certaines choses qui se sont produites dans mon passé

-pour dire la vérité sur certaines choses qui se sont produites dans mon passé

-pour pouvoir justifier certaines choses que j'ai pu faire dans le passé

-pour me confesser sur centaines choses

-car j'ai envie de m'exprimer sur des choses qui me tiennent particulièrement à cœur

Flatter l'ego

-parce qu'il y a des aspects de ma vie qui mérite d'être connus

-pour montrer comment je suis devenue la personne que je suis aujourd'hui

-pour parler de moi et des choses que j'ai pu faire dans ma vie

-parce qu'il y a des choses que j'ai faites et qui méritent plus de reconnaissance

* pour parler des choses intéressantes que j’ai pu faire dans ma vie

* pour faire connaître des choses que j'ai faites et dont je suis fier(ère)

*pour faire connaître des aspects de moi que les autres ne connaissent pas forcément

*pour montrer aux personnes qui l'ignorent qui je suis réellement

* pour montrer d'où je viens et comment je suis devenue la personne que je suis aujourd'hui

Transmettre

- pour éviter aux plus jeunes de faire les mêmes erreurs que j'ai pu commettre

-pour transmettre aux plus jeunes certaines valeurs que je juge essentielles

-pour faire connaître aux plus jeunes leurs racines

-par la transmission de mon vécu et de mes expériences, j'essaie d'être utile aux autres

-parce que j'essaie de faire comprendre aux plus jeunes ce qu'il faut faire ou ne pas

faire

*pour retracer l'histoire de ma famille

*pour faire connaître aux plus jeunes les membres de la famille aujourd'hui disparus

* pour transmettre des valeurs familiales

*pour répondre aux désirs des plus jeunes membres de la famille de connaître leurs

racines 
Annexe A2 : Caractéristiques socio-démographiques des données quantitatives

\begin{tabular}{|l|c|c|}
\hline & $\begin{array}{c}1^{\text {ere }} \text { collecte } \\
202 \text { individus }\end{array}$ & $\begin{array}{c}2^{\text {nd }} \text { collecte } \\
508 \text { individus }\end{array}$ \\
\hline Age & & 68,80 \\
\hline Age moyen & 71,28 & 6,84 \\
\hline Ecart-type & 8,60 & 60 \\
\hline Minimum & 60 & 94 \\
\hline Maximum & 96 & $36 \%$ \\
\hline Sexe & & $64 \%$ \\
\hline Hommes & $44 \%$ & $8,7 \%$ \\
\hline Femmes & $56 \%$ & $22,1 \%$ \\
\hline Niveau d'études & & $13 \%$ \\
\hline Aucun diplôme & $22,8 \%$ & $22,7 \%$ \\
\hline Certificat d'études & $25,4 \%$ & $19,7 \%$ \\
\hline CAP / BEP & $10,9 \%$ & $13,8 \%$ \\
\hline Niveau Bac & $11,4 \%$ & \\
\hline Bac +1 à +3 & $12,4 \%$ & $12,2 \%$ \\
\hline Bac +4 et plus & $17,1 \%$ & $12 \%$ \\
\hline Nombre d'enfants & & $73,8 \%$ \\
\hline Pas d'enfant & $3,1 \%$ & $2 \%$ \\
\hline 1 enfant & $13,9 \%$ & \\
\hline 2 à 4 enfants & $72,7 \%$ & \\
\hline 5 enfants et plus & $10,3 \%$ & \\
\hline
\end{tabular}




\section{Annexe A3 : L'échelle des motivations au récit de vie chez la personne âgée}

\section{Consigne :}

Une histoire de vie consiste à raconter une histoire à partir de vos souvenirs. Veuillez indiquer si les propositions suivantes vous concernent ou non (pas du tout d'accord, pas d'accord, cela dépend, d'accord, tout à fait d'accord).

\section{J'aimerais écrire une histoire de vie ...}

\section{Ne pas être oublié $(\alpha=\mathbf{0 , 8 3})$}

pour que mes proches se rappellent de moi après ma mort pour dire à mes proches ce que j'aimerais que l'on retienne de moi pour que l'on se souvienne de ce que j'ai fait après ma mort

\section{Témoigner $(\alpha=\mathbf{0 , 8 3})$}

pour laisser des traces sur la façon dont nous vivons, afin que les générations futures puissent reconstituer notre mode de vie pour laisser un témoignage car cela permet de créer une sorte de patrimoine qu'on lègue aux générations futures

pour préserver certains aspects de ma culture qui sont en train de disparaître pour noter quelque part certaines choses du passé qui ne doivent pas être perdues

\section{Partager $(\alpha=0,79)$}

pour créer une forme de complicité avec les lecteurs

pour élargir le cercle de mes connaissances

pour créer des liens avec certaines personnes

\section{Réparer l'ego $(\alpha=0,81)$}

pour m'aider à clarifier une période de ma vie

pour pouvoir donner ma version des faits sur certaines choses qui se sont produites dans mon passé pour dire la vérité sur certaines choses qui se sont produites dans mon passé pour pouvoir justifier certaines choses que j'ai pu faire dans le passé

\section{Flatter l'ego $(\alpha=\mathbf{0 , 8 0})$}

pour parler des choses intéressantes que j'ai pu faire dans ma vie pour faire connaître des choses que j'ai faites et dont je suis fier(ère) pour faire connaître des aspects de moi que les autres ne connaissent pas forcément

\section{Transmettre $(\alpha=0,81)$}

pour faire connaître aux plus jeunes les membres de la famille aujourd'hui disparus pour transmettre des valeurs familiales pour répondre aux désirs des plus jeunes membres de la famille de connaître leurs racines 
Tableau $n^{\circ} 1$ - Les étapes de la construction et de la validation de l'échelle de mesure

\begin{tabular}{|c|c|}
\hline Etapes réalisées & Principaux résultats \\
\hline $\begin{array}{l}1 \text { - Spécification du } \\
\text { domaine du construit }\end{array}$ & $\begin{array}{l}\text { - Il existe } 6 \text { types de motivations au récit de vie, issues de la } \\
\text { littérature, confirmées par analyse qualitative (non développée ici) } \\
\text { - Ces indicateurs sont des effets du construit. Le paradigme de } \\
\text { Churchill est adapté }\end{array}$ \\
\hline $\begin{array}{l}2 \text { - Création et validation } \\
\text { des items }\end{array}$ & $\begin{array}{l}\text { - } 16 \text { entretiens semi-directifs (non développés ici) } \\
\text {-77 items générés à partir de la littérature et des entretiens } \\
\text { qualitatifs } \\
\text {-36 items retenus par un panel d'experts (validité de contenu) }\end{array}$ \\
\hline $\begin{array}{l}3 \text { - Première collecte de } \\
\text { données et purification } \\
\text { des items }\end{array}$ & $\begin{array}{l}\text {-202 réponses } \\
-22 \text { items éliminés par } 6 \text { ACP (rotation oblique) } \\
\text {-Les dimensions transmettre et flatter l'ego n'apparaissent pas : } 9 \\
\text { nouveaux items générés représentant ces dimensions }\end{array}$ \\
\hline $\begin{array}{l}4-\text { Seconde collecte de } \\
\text { données et émergence } \\
\text { d'une structure } \\
\text { factorielle }\end{array}$ & $\begin{array}{l}\text { - } 508 \text { réponses } \\
\text { - } 3 \text { items supprimés par } 3 \text { ACP } \\
\text {-20 items s'agrègent sur } 6 \text { dimensions : flatter l'ego }(\alpha=0,80) ; \\
\text { réparer l'ego }(\alpha=0,81) ; \text { ne pas être oublié }(\alpha=0,83) ; \text { partager } \\
(\alpha=0,79) ; \text { transmettre }(\alpha=0,81) \text { et témoigner }(\alpha=0,83)\end{array}$ \\
\hline $\begin{array}{l}5 \text { - Validation de la } \\
\text { structure factorielle }\end{array}$ & $\begin{array}{l}\text {-Analyse factorielle confirmatoire } \\
\text {-Estimation par ML. Statistiques « robustes » qui corrigent } \\
\text { l'absence de normalité des données } \\
-\chi^{2}=340 ; \text { ddl }=155(p=0,00) ; C F I=0,95 ; \text { RMSEA }=0,05 \\
\text { - Tous les modèles alternatifs testés produisent de moins bons } \\
\text { indices d'ajustements. } \\
\text {-Structure factorielle validée }\end{array}$ \\
\hline $\begin{array}{l}6 \text { - Fiabilité, validités } \\
\text { convergente et } \\
\text { discriminante du modèle } \\
\text { de mesure }\end{array}$ & $\begin{array}{l}\text {-Tous les } \rho>0,80 \text { (fiabilité) } \\
\text {-Coefficients structurels liés significativement (test } \mathrm{z}>1,96) \text { et } \rho_{\mathrm{vc}}> \\
0,5 \text { (validité convergente) } \\
\text {-Les corrélations entre deux construits élevées au carré sont } \\
\text { inférieures à leurs } \rho_{\mathrm{vc}} \text { (validité discriminante) }\end{array}$ \\
\hline $\begin{array}{l}7 \text { - Analyse factorielle } \\
\text { de second ordre, validité } \\
\text { nomologique }\end{array}$ & $\begin{array}{l}\text {-Existence d'un facteur de second ordre }\left(\chi^{2}=381 ; \mathrm{ddl}=164\right. \\
(\mathrm{p}=0,00) ; \mathrm{CFI}=0,95 ; \mathrm{RMSEA}=0,05) ; \text { coef. structurels }>0,70 ; \\
\text { représentant la motivation au récit de vie « globale » } \\
\text {-La corrélation entre cette motivation globale et l'intention } \\
\text { effective d'écrire sa vie est de } 0,5 \text { (validité nomologique) }\end{array}$ \\
\hline
\end{tabular}


Tableau $\mathrm{n}^{\circ} \mathbf{2}$ - Structure factorielle finale des 20 items

\begin{tabular}{|c|c|c|c|c|c|c|c|c|}
\hline & & & & & & & & \\
\hline & & Communalité & Facteur 1 & Facteur 2 & Facteur 3 & Facteur 4 & Facteur 5 & Facteur 6 \\
\hline $\begin{array}{l}\text { Ne pas être } \\
\text { oublié } \\
(\alpha=\mathbf{0 , 8 3})\end{array}$ & \begin{tabular}{|l|} 
pour que mes proches se rappellent de moi après ma mort \\
pour dire à mes proches ce que j'aimerais que l'on retienne de moi \\
pour que l'on se souvienne de ce que j'ai fait après ma mort
\end{tabular} & $\begin{array}{l}0,72 \\
0,71 \\
0,79 \\
\end{array}$ & $\begin{array}{l}0,77 \\
0,69 \\
0,82 \\
\end{array}$ & & & & & \\
\hline $\begin{array}{l}\text { Témoigner } \\
(\alpha=\mathbf{0 , 8 3})\end{array}$ & $\begin{array}{l}\text { pour laisser des traces sur la façon dont nous vivons, afin que les générations futures } \\
\text { puissent reconstituer notre mode de vie } \\
\text { pour laisser un témoignage car cela permet de créer une sorte de patrimoine qu'on } \\
\text { lègue aux générations futures } \\
\text { pour préserver certains aspects de ma culture qui sont en train de disparaître } \\
\text { pour noter quelque part certaines choses du passé qui ne doivent pas être perdues }\end{array}$ & $\begin{array}{l}0,70 \\
0,74 \\
0,63 \\
0,66\end{array}$ & & $\begin{array}{l}0,71 \\
0,79 \\
0,60 \\
0,70\end{array}$ & & & & \\
\hline $\begin{array}{l}\text { Partager } \\
(\alpha=0,79)\end{array}$ & $\begin{array}{l}\text { pour créer une forme de complicité avec les lecteurs } \\
\text { pour élargir le cercle de mes connaissances } \\
\text { pour créer des liens avec certaines personnes }\end{array}$ & $\begin{array}{l}0,65 \\
0,73 \\
0,74\end{array}$ & & & $\begin{array}{l}0,72 \\
0,81 \\
0,86\end{array}$ & & & \\
\hline $\begin{array}{l}\text { Réparer l'ego } \\
\qquad(\alpha=\mathbf{0 , 8 1})\end{array}$ & $\begin{array}{l}\text { pour m'aider à clarifier une période de ma vie } \\
\text { pour pouvoir donner ma version des faits sur certaines choses qui se sont produites } \\
\text { dans mon passé } \\
\text { pour dire la vérité sur certaines choses qui se sont produites dans mon passé } \\
\text { pour pouvoir justifier certaines choses que j'ai pu faire dans le passé }\end{array}$ & $\begin{array}{l}0,58 \\
0,72 \\
0,74 \\
0,67\end{array}$ & & & & $\begin{array}{l}0,68 \\
0,75 \\
0,81 \\
0,64\end{array}$ & & \\
\hline $\begin{array}{l}\text { Flatter l'ego } \\
\qquad(\alpha=\mathbf{0 , 8 0})\end{array}$ & $\begin{array}{l}\text { pour parler des choses intéressantes que j'ai pu faire dans ma vie } \\
\text { pour faire connaître des choses que j'ai faites et dont je suis fier(ère) } \\
\text { pour faire connaître des aspects de moi que les autres ne connaissent pas forcément }\end{array}$ & $\begin{array}{l}0,72 \\
0,79 \\
0,71\end{array}$ & & & & & $\begin{array}{l}0,82 \\
0,75 \\
0,58\end{array}$ & \\
\hline $\begin{array}{l}\text { Transmettre } \\
\qquad(\alpha=\mathbf{0 , 8 1})\end{array}$ & $\begin{array}{l}\text { pour faire connaître aux plus jeunes les membres de la famille aujourd'hui disparus } \\
\text { pour transmettre des valeurs familiales } \\
\text { pour répondre aux désirs des plus jeunes membres de la famille de connaître leurs } \\
\text { racines }\end{array}$ & $\begin{array}{l}0,74 \\
0,75 \\
0,76\end{array}$ & & & & & & $\begin{array}{l}0,86 \\
0,83 \\
0,82\end{array}$ \\
\hline & \begin{tabular}{|l|l|} 
Valeur propre \\
\end{tabular} & & 8,2 & 1,74 & 1,24 & 1,10 & 1,07 & 0,93 \\
\hline & $\%$ de variance expliquée & $71 \%$ & $41 \%$ & $9 \%$ & $6 \%$ & $5 \%$ & $5 \%$ & $5 \%$ \\
\hline
\end{tabular}


Tableau $n^{\circ} 3$ - Evaluation de la fiabilité et de la validité convergente

\begin{tabular}{|c|c|c|c|}
\cline { 2 - 4 } \multicolumn{1}{c|}{} & Fiabilité & \multicolumn{2}{c|}{$\begin{array}{c}\text { Validité } \\
\text { convergente }\end{array}$} \\
\hline Dimensions & $\rho$ & tests $\mathrm{z}$ & $\rho_{\mathrm{vc}}$ \\
\hline Ne pas être oublié & 0,82 & $\mathrm{z}>20$ & 0,63 \\
\hline Témoigner & 0,84 & $\mathrm{z}>16$ & 0,57 \\
\hline Partager & 0,80 & $\mathrm{z}>16$ & 0,57 \\
\hline Réparer l'ego & 0,82 & $\mathrm{z}>13$ & 0,53 \\
\hline Flatter l'ego & 0,81 & $\mathrm{z}>13$ & 0,59 \\
\hline Transmettre & 0,83 & $\mathrm{z}>16$ & 0,61 \\
\hline
\end{tabular}


Tableau n ${ }^{\circ} 4$ - Evaluation de la validité discriminante et corrélation entre les construits

\begin{tabular}{|l|c|c|c|c|c|c|}
\hline & $\begin{array}{c}\text { Ne pas être } \\
\text { oublié }\end{array}$ & Témoigner & Partager & $\begin{array}{c}\text { Réparer } \\
\text { l'ego }\end{array}$ & $\begin{array}{c}\text { Flatter } \\
\text { l'ego }\end{array}$ & Transmettre \\
\hline Ne pas être oublié & 0,79 & & & & & \\
\hline Témoigner & 0,67 & 0,75 & & & & \\
\hline Partager & 0,62 & 0,63 & 0,75 & & & \\
\hline Réparer l'ego & 0,70 & 0,56 & 0,65 & 0,73 & & \\
\hline Flatter l'ego & 0,69 & 0,54 & 0,62 & 0,69 & 0,77 & \\
\hline Transmettre & 0,58 & 0,69 & 0,50 & 0,55 & 0,51 & 0,78 \\
\hline
\end{tabular}


Tableau $n^{\circ} 5$ - Analyse factorielle de second ordre

\begin{tabular}{|c|c|}
\hline $\begin{array}{c}\text { Facteurs de premier } \\
\text { ordre }\end{array}$ & $\begin{array}{c}\text { Loadings } \\
\text { (standardisés) }\end{array}$ \\
\hline Ne pas être oublié & 0,853 \\
\hline Témoigner & 0,780 \\
\hline Partager & 0,768 \\
\hline Réparer l'ego & 0,813 \\
\hline Flatter l'ego & 0,779 \\
\hline Transmettre & 0,713 \\
\hline
\end{tabular}


BIBLIOGRAPHIE

d'Almeida N. et Merran-Ifrah S. (2005), Le récit patronal, la tentation autobiographique, Revue Française de Gestion, 159, 109-122

Argentin L. (2006), Parler de son temps: récits de vie et ateliers d'écritures, Soins Gérontologie, 57, 38-42

Bagozzi R.P (2007), On the Meaning of Formative Measurement and How it Differs from Reflective Measurement: Comment on Howell, Breivik, and Wilcox (2007), Psychological Methods, 12, 2, 229-237

Bandura A. (1986), Social foundations of thought and action: A social cognitive theory, Prentice-Hall (Englewood Cliffs N.J.)

Barreau A. (1992), Quelle mort pour demain? Essai d'anthropologie prospective, L'Harmattan

Belk R. (1988), Possessions and the Extended Self, Journal of Consumer Research, 15, 2, 139168

Bentler P.M. (2005), EQS 6 structural equations program manual, Encino, CA: Multivariate Software (www.mvsoft.com)

Bergadàa M. (2004), Évolution de l'épistémè économique et sociale: proposition d'un cadre de morale, de déontologie, d'éthique et de responsabilité pour le marketer, Recherche et Applications en Marketing, 19,1, 55-72

Bergadaà M. et Urien B. (2008), La personne âgée : son attitude face à l'idée de mort, le cadre temporel de son action et son sens de l'objet, «Rupture, finitude, mort et Management» Seconde conférence internationale sur le temps de Brest, 25-26 juin

Bergkvist L. et Rossiter J.R (2008), Comparaison des validités prédictives des mesures d'un même construit des échelles mono-item et des échelles multi-items, Recherche et Applications en Marketing, 23, 1, 81-96 
Billé M. (2002), A quoi servent les grands-parents? Des grands-parents pour introduire au « sacré », Dialogue, 158, 3-10

Billé M. (2006), Le lien social au cœur de la prévention, Le traitement social de la vieillesse, La gérontologie en actes, L'Harmattan, 71-84

Blanchard F. (2006), Rejouer les événements de vie du grand âge, La revue Francophone de Gériatrie et de Gérontologie, 123, 128-132

Bollen K.A. (2007), Interpretational Confounding is due to misspecification, Not to type of indicator: Comment on Howell, Breivik and Wilcox (2007), Psychological Methods, 12, 2, $219-228$

Bresay C. (2004), Vieillir, Etudes sur la mort thanatologie, mourir âgé en institution, 126, 3742

Butler R.N. (1963), The Life Review: an interpretation of reminiscence in the age, Psychiatry, $26,65-75$

Byrne B.M. (2006), Structural equation modeling with EQS: Basic concepts, applications, and programming, Lawrence Erlbraum associates Publishers, London, $2^{\text {nd }}$ edition

Cardon D. et Delaunay-Teterel H. (2006), La production de soi comme technique relationnelle. Un essai de typologie des blogs par leurs publics, Réseaux, 24, 138, 15-71

Carron J-P. (2002), Ecriture et identité, pour une poétique de l'autobiographie, Edition Ousia, Bruxelles

Castelnuovo-Tedesco P. (1980), Reminiscence and nostalgia: The Pleasure and pain of remembering, In Greenspan S.I. et Pollock G.H. (coord.), The course of life: Psychoanalytic contributions toward understanding personality development (Vol III: Adulthood and the aging Process), Washington DC: US Department of Health and Human Services, pp 115-127

Churchill G.A. (1979), A paradigm for Developing Better Measures of Marketing Constructs, Journal of Marketing Research, 16, 64-73 
Crié D. (2005), De l'usage des modèles de mesure réflexifs ou formatifs dans les modèles d'équations structurelles, Recherche et Applications en Marketing, 20, 2, 5-27

Cristini C. et Ploton L. (2009), Mémoire et autobiographie, Gérontologie et Société, 130, 75-95

Curasi C.F. (2006), Maybe it is your father's oldsmobile: the construction and preservation of family identity through the transfer of possessions, Advances in Consumer Research, 33, 8286

Curasi C.F., Price L.L. et Arnould E.J. (2004), How individuals' cherished possessions become families' inalienable wealth, Journal of Consumer Research, 31, 3, 609-623

Delory-Momberger C. (2005), Histoire de vie et recherche biographique en éducation, Economica

Diamantopoulos A. (2006), The error term in formative measurement models: Interpretation and modelling implication, Journal of Modelling in Management, 1, 7-17

Diamantopoulos A., Riefler P. et Roth K.P. (2008), Advancing formative measurement models, Journal of Business Research, 61, 1203-1218

Diamantopoulos A. et Winklhofer H.M. (2001), Index Construction with Formative Indicators: An Alternative to Scale Development, Journal of Marketing Research, 38, 269-277

DiStefano C. (2002), The impact of categorization with confirmatory factor analysis, Structural Equation Modeling: A multidisciplinary Journal, 9, 327-346

Epp A.M. et Arnould E.J. (2006), Enacting the family legacy: How family themes influence consumption behaviour, Advances in Consumer Research, 33, 82-86

Erikson E.H. (1959), Identity and the life cycle: Selected papers, Psychological Issues, 1, 50100

Florian V. et Kravetz S (1983), Fear of personal death: Attribution, structure, and relation to religious belief, Journal of Personality and Social Psychology, 44, 3, 600-607 
Florian V. et Snowden L.R. (1989), Fear of Personal Death and Positive Life Regard, Journal of Cross-Cultural Psychology, 20, 1, 64-79

Fornell C. et Larker D.F. (1981), Evaluating structural equations models with unobservable variable and mesurement error, Journal of Marketing Research, 18, 39-50

Franke G.R. , Preacher K.J. et Rigdon E.E. (2008), Proportional structural effects of formative indicators, Journal of Business Research, 61, 1229-1237

Fry M.L. (1990), Cross-cultural comparisons of aging, In Ferraro K.F. (Ed), Gerontology: Perspectives and Issues, New York, NY, US, Springer Publishing Co, 129-146

Goldberg A. (2009), Réaliser une boîte à souvenir, Gérontologie et société, 130, 155-170

Guiot D. (2006), Un cadre d'analyse du processus de vieillissement et de son influence sur le comportement d'achat du consommateur âgé, Recherche et Applications en Marketing, 27, 1, $57-79$

Gusdorf G. (1991), Lignes de vie - Les écritures du moi (Tome 1), Editions Odile Jacob

Haber D. (2006), Life Review: Implantation, Theory, Research and Therapy, International Journal of Aging and Human Development, 63, 2, 153-171

Hair J.F. Jr., Black W.C., Babin J.B., Anderson R.E., Tatham R.L. (2008), Multivariate data analysis, $7^{\text {th }}$ edition, Pearson, Prentice Hall

Howell R.D., Breivik E. et Wilcox J.B. (2007a), Reconsidering Formative Measurement, Psychological Methods, 12, 2, 205-218

Howell R.D., Breivik E. et Wilcox J.B. (2007b), Is Formative Measurement really Measurement? Reply to Bollen (2007) and Bagozzi (2007), Psychological Methods, 12, 2, 238-245

Hubier S. (2003), Littératures intimes: les expressions du moi, de l'autobiographie à l'autofiction, A. Colin 
Janvier M. (2007), Atelier d'écriture et personnes âgées, Aux vendanges de la vie, Chronique Sociale, Lyon

Jarvis B.C., Mackenzie S.B. et Podsakoff P.M. (2003), Critical review of construct indicators and measurement model misspecification in marketing and consumer research, Journal of Consumer Research, 30, 2, 199-218

Jolibert A. et Jourdan P. (2006), Marketing research, Méthodes de recherche et d'études en marketing, Dunod

Kaiser H. et Rice J (1974), «Little Jiffy Mark 4 », Educational and Psychology Measurement, $34,111-117$

Katz S. et Marshall B. (2003), New sex for old: lifestyle, consumerism, and the ethics of aging well, Journal of Aging Studies, 17, 3-16

Lalive d'Epinay C. et Cavalli S. (2009), Mémoire de l'histoire et appartenance générationnelle des personnes âgées, Gérontologie et société, 130, 127-144

Larcher H. (1971), Mnémothèques pour l'An 2000, Bulletin de la Société de Thanatologie, 17, $1-17$

Lecarme J. et Lecarme-Tabone E. (1999), L'autobiographie, $2^{\text {nd }}$ édition, Armand Colin, Paris

Lejeune P. (1975), Le pacte autobiographique, édition du seuil, Paris

Lejeune P. (2005), Signes de vie : Le pacte autobiographique 2, Edition du Seuil, Paris

Madelénat D. (2008), Moi, biographe : m'as-tu vu ?, Revue de littérature comparée, 325, 95108

Martin Sanchez M-O. (2003), Discours oraux, discours écrits, les seniors et l'écriture de soi, Gérontologie et Société, 106, 97-109

May G. (1979), L'autobiographie, PUF, Paris 
McAdams D.P. et de St. Aubin E. (1992), A theory of generativity and its assessment through self-report, behavioural acts, and narrative themes in autobiography, Journal of Personality and Social Psychology, 62, 1003-1015

Meredith G. et Schewe C.D. (1994), Cohort effects, Marketing Management, Winter, 3, 3-25

Mezred D., Petignet V., Fort I., Blaison C., Gana K. (2006), La réminiscence: concept, fonctions et mesures. Adaptation française de la Reminiscence Functions Scale, Les Cahiers Internationaux de Psychologie Sociale, 71, 3-14

Miraux J-P. (2005), L'autobiographie, écriture de soi et sincérité, Armand Colin

Pineau G. et Le Grand J-L. (1993), Les histoires de vie, PUF, collection «Que sais-je », $2^{\text {nd }}$ édition 2002

Podaskoff P.M., MacKenzie S.B, Podaskoff N.P et Lee J.Y (2003), The mismeasure of man(agement) and its implications for leadership research, Leadership Quarterly, 14, 615-656

Préel B. (2000), Le choc des générations, Editions la Découverte, Paris

Price L.L., Arnould E.J. et Curasi C.F. (2000), Older consumers' disposition of special possessions, Journal of Consumer Research, 27, 2, 179-201

Pudal B. (2000), Ecritures non professionnelles et prises de parole, Les Actes de Lecture, 69, 93-96

Ribes G., Sagne A., Gaucher J. et Ploton L. (2009), Mémoire générationnelle et identité, Gérontologie et Société, 130, 145-153

Rioul R. (2003), Le désir autobiographique, Conférence prononcée le 7 mars dans le cadre de l’Université du Temps Libre, Université Marc-Bloch, Strasbourg

Roche A. (2007), Le modèle autobiographique est-il exportable ? Exemples au Maghreb, in Simonet-Tenant F. (ed), Le propre de l'écriture de soi, Paris : Téraèdre, 156-164

Ross H.K (1990), Lesson of life, Geriatric Nursing, 11, 274-275 
Rossiter J.R. (2002), The C-OAR-SE procedure for scale development in marketing, International Journal of Research in Marketing, 19, 305-335

Satorra A. et Bentler P.M. (1988), Scaling corrections for chi square statistics in covariance structure analysis, American Statistical Association, Proceedings of the Business and Economic Sections, $308-313$

Steenkamp J.B. et Van Trijp H.C. (1991), The use of LISREL in validating Marketing construct, International Journal of Research in Marketing, 8, 283 - 289

Tarman V.I. (1988), Autobiography: The negociation of a lifetime, International Journal of Aging and Human Development, 27, 3, 171-191

Trueman I. et Parker J. (2006), Exploring community nurses' perceptions of life review in palliative care, Journal of Clinical Nursing, 15, 197-207

Urien B. (2003), L'anxiété face à la mort et son influence sur le comportement du consommateur, Recherche et Applications en Marketing, 18, 4, 23-41

Urien B. et Guiot D. (2007), Attitude face à la mort et comportement d'ajustement des consommateurs âgés : Vers l'élaboration d'une réponse marketing, Décisions Marketing, 46, 23-35

Urien B. et Kilbourne W. (2007), Generativity and its Relationship to Eco-Friendly Consumption Behavior, American Marketing Association (AMA) Winter Marketing Educator's Conference, San Diego, Californie, USA

Urien B. et Kilbourne W. (2011), On the Role of Generativity and Self-Enhancement Values in Eco-Friendly Behavioural Intentions and Environmentally Responsible Consumption Behaviour, Psychology and Marketing, 28, 1, 69-90

Vallerand, R.J (1997), toward a hierarchical model of intrinsic and extrinsic motivation, in Zanna M.P. (coord.), Advances in experimental social psychology, 29, 271-360, New York : Academic Press . 
Vallerand, R.J. (2000). Deci and Ryan's self-determination theory: A view from the hierarchical model of intrinsic and extrinsic motivation. Psychological Inquiry, 11 , 312-318.

Vallerand, R.J., \& Miquelon, P. (2009). Le Modèle Hiérarchique: Une analyse intégrative des déterminants et conséquences de la motivation intrinsèque et extrinsèque. In Joule R-V (coord.), Bilans et perspectives en Psychologie Sociale. Grenoble, France: Presses de l’Université de Grenoble.

Vercauteren R. et Hervy B. (2003), L'animation dans les établissements pour personnes âgées, Manuel des pratiques professionnelles, Pratiques gérontologiques, Eres édition

Wallendorf M. et Arnould E.J. (1988), "My favourite things": a cross-cultural inquiry into object attachment, possessiveness, and social linkage, Journal of Consumer Research, 14, 4, $531-547$

Wapner S., Dermick J. et Redondo J.P. (1990), Cherished Possessions and Adaptation of Older People to Nursing Homes, International Journal of Aging and Human Development, 31, 3, 219-236

Webster J.D. (1993), Construction and validation of the reminiscence Function Scale, Journal of Gerontology: Psychological Sciences, 48, 256-262

Wilcox J.B, Howell R.D et Breivik E. (2008), Questions about formative measurement, Journal of Business Research, 61, 1219-1228

Zanone D. (2006), L'autobiographie, Ellipses

Zarca B. (2009), Triple démarche pour une transformation de soi. Psychanalyse, socio-analyse et autobiographie, Le coq-héron, 198, 118-130

Zemke R., Raines C. et Filipczak B. (2000), Generations at work: Managing the clash of veterans, boomers, Xers and nexers in your workplace, New York: Amacom 